\title{
First thia-Diels-Alder reactions of thiochalcones with 1,4-quinones
}

\author{
Grzegorz Mlostoń ${ }^{* 1}$, Katarzyna Urbaniak ${ }^{1}$, Paweł Urbaniak ${ }^{2}$, Anna Marko ${ }^{1}$, \\ Anthony Linden ${ }^{3}$ and Heinz Heimgartner ${ }^{3}$
}

\author{
Full Research Paper \\ Address: \\ ${ }^{1}$ Department of Organic and Applied Chemistry, University of Łódź, \\ Tamka 12, PL 91-403 Łódź, Poland, ${ }^{2}$ Department of Inorganic and \\ Analytical Chemistry, University of Łódź, Tamka 12, PL 91-403 Łódź, \\ Poland and ${ }^{3}$ Department of Chemistry, University of Zurich, \\ Winterthurerstrasse 190, CH-8057 Zurich, Switzerland \\ Email: \\ Grzegorz Mlostoń* - grzegorz.mloston@chemia.uni.lodz.pl \\ * Corresponding author \\ Keywords: \\ hetero-Diels-Alder reactions; quinone dyes; quinones; sulfur \\ heterocycles; thiochalcones
}

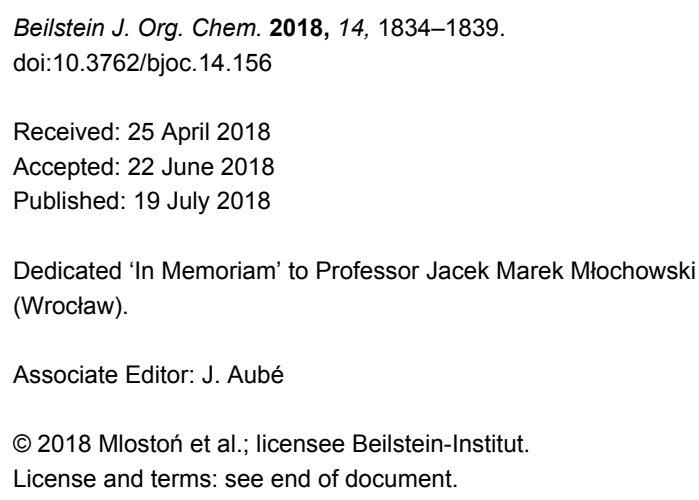

\section{Abstract}

Aryl and hetaryl thiochalcones react smoothly with 1,4-quinones in THF solution at $60{ }^{\circ} \mathrm{C}$ yielding the corresponding fused $4 H$-thiopyrans after spontaneous dehydrogenation of the initially formed [4+2] cycloadducts. In general, the yields of the isolated products were high. With 5-chloro-10-hydroxy-1,4-anthraquinone, the thia-Diels-Alder reaction occurred with complete regioselectivity. In the case of the reaction of vitamin $\mathrm{K}_{3}$ (menadione) with diphenylthiochalcone, the initial cycloadduct was isolated in $37 \%$ yield.

\section{Introduction}

Hetero-Diels-Alder reactions are considered to be a powerful methodology widely explored for the synthesis of six-membered heterocycles $[1,2]$ with numerous applications for the construction of complex molecules including naturally occurring products [3,4], drugs [5,6], agrochemicals [7], etc. In addition, asymmetric hetero-Diels-Alder reactions are of current interest [8-10]. Whereas aza- and oxa-Diels-Alder reactions are frequently applied, thia-Diels-Alder reactions are rarely reported. However, aryl and hetaryl thioketones are known to react as 'superdienophiles', thereby yielding the corresponding 3,6-dihydro-2H-thiopyrans [11-14].

Despite the fact that thiochalcones exist in solution as mixtures of dimers $[15,16]$, they enter into cycloaddition reactions not only as heterodienes [17-19], but also as heterodipolarophiles [15]. In two recent publications we reported new thiaDiels-Alder reactions of aryl, hetaryl and ferrocenyl-substituted thiochalcones with acetylenic dienophiles, which lead to 
the corresponding $4 H$-thiopyrans in a regioselective manner $[16,20]$.

In cycloaddition chemistry, 1,4-quinones are applied widely both as dipolarophiles and dienophiles. In the case of $[3+2]$ cycloadditions, reactions can occur chemoselectively with either the $\mathrm{C}=\mathrm{O}$ or the $\mathrm{C}=\mathrm{C}$ unit [21-23]. On the other hand, reactions with diverse 1,3-dienes and heterodienes generally occur at the $\mathrm{C}=\mathrm{C}$ group [24-26]. To the best of our knowledge, thiaDiels-Alder reactions of 1,4-quinones with thiochalcones have not yet been reported.

In the present study, thia-Diels-Alder reactions of aryl and hetaryl thiochalcones with selected 1,4-quinones, such as 1,4benzoquinone, 1,4-naphthoquinone, and 1,4-anthraquinone were investigated as a route to novel $4 H$-thiochromene-5,8dione derivatives.

\section{Results and Discussion}

Aryl and hetary lthiochalcones $\mathbf{1 a}-\mathbf{d}$ are easily obtained by treatment of the corresponding chalcones with Lawesson's reagent in THF solution [15]. Along with the commercially available 1,4-benzoquinone (2a) and 1,4-naphthoquinone (2b), 1,4-anthraquinone (2c) and 5-chloro-10-hydroxy-1,4anthraquinone (2d) were prepared from quinizarine according to known procedures $[27,28]$.

First experiments were performed with $\mathbf{2} \mathbf{b}$ and thiochalcones $\mathbf{1 a}-\mathbf{d}$ in THF solutions at $60{ }^{\circ} \mathrm{C}$ by starting with equimolar amounts of substrates. After $2 \mathrm{~h}$, completion of the reaction was confirmed by TLC, and, after typical workup, products 4 were obtained as colored solids in high yields (Scheme 1).
The ${ }^{1} \mathrm{H}$ NMR analysis revealed that the initially formed [4 + 2] cycloadducts 3 underwent spontaneous oxidation under the reaction conditions. The structures of type 4 were confirmed by spectroscopic methods and elemental analysis. For example, the ${ }^{1} \mathrm{H}$ NMR spectrum of $\mathbf{4 a}$ showed two doublets at 5.46 and $6.35 \mathrm{ppm}$ with $J=6.4 \mathrm{~Hz}$ attributed to $\mathrm{H}-\mathrm{C}(4)$ and $\mathrm{H}-\mathrm{C}(3)$, respectively. In the ${ }^{13} \mathrm{C}$ NMR spectrum, the $\mathrm{sp}^{3}-\mathrm{C}(4)$ atom absorbs at $40.9 \mathrm{ppm}$ and the signals for the two $\mathrm{C}=\mathrm{O}$ groups were found at 180.8 and $181.4 \mathrm{ppm}$, respectively.

In the second series of experiments, thiochalcones $\mathbf{1 a}-\mathbf{d}$ were subjected to reaction with the symmetrical 1,4-anthraquinone (2c) and its non-symmetrically substituted derivative $\mathbf{2 d}$. In analogy to the reactions with $\mathbf{2 b}$, the expected products $\mathbf{4 e -}-\mathbf{h}$ were isolated in all cases as stable colored solids in high yields (Scheme 2).

The reactions of thiochalcones $\mathbf{1 a}-\mathbf{d}$ with $\mathbf{2 d}$ require a brief discussion. In these cases, the formation of two regioisomeric cycloadducts could be expected, but the ${ }^{1} \mathrm{H}$ NMR analysis of the crude products showed that only one product was present in each case and, therefore, the studied [4 +2] cycloaddition reactions occurred with complete regioselectivity. Based on the assumption that the nucleophilic S-atom of the thiochalcone attacks the more electrophilic $\mathrm{C}$-atom, we postulate that compounds $\mathbf{4} \mathbf{i}-\mathbf{l}$ and not their isomers $\mathbf{5 a}-\mathbf{d}$ are formed in these reactions. This assumption is supported by the intramolecular H-bonding, which enhances the electrophilicity of C(3) in the dienophile 2d. Finally, the structure of $\mathbf{4 k}$ was established by $\mathrm{X}$-ray crystallography (Figure 1). Remarkably, the presence of $\mathrm{OH}$ and $\mathrm{Cl}$ substituents in products $\mathbf{4} \mathbf{i}-\mathbf{l}$ results in a bathochromic shift of UV-vis absorptions.

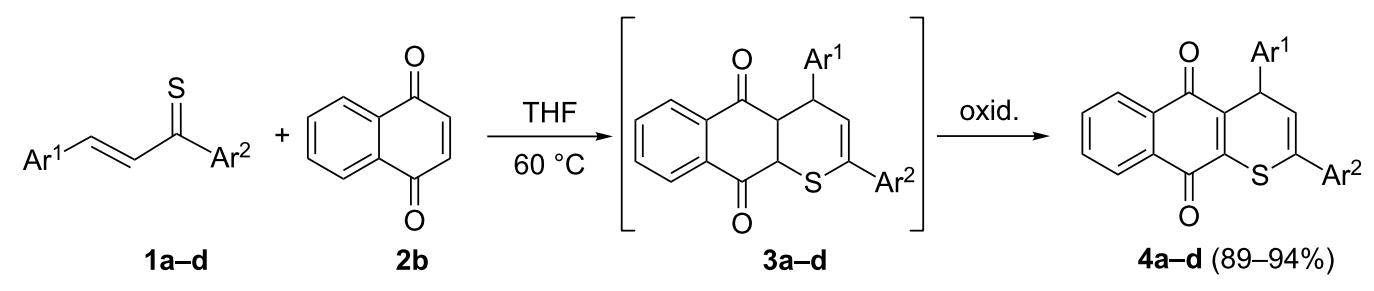

a: $\mathrm{Ar}^{1}=A r^{2}=\mathrm{Ph}$

b: $A r^{1}=\left\langle{ }_{S} \|_{-}, A r^{2}=P h\right.$

c: $\operatorname{Ar}^{1}=P h, A r^{2}=\left\langle{ }_{S}\right.$

d: $A r^{1}=A r^{2}=\left\langle{ }_{S}\right\rangle^{\prime}$ 
<smiles>[Al]/C=C/C(=S)Br</smiles>

1a-d

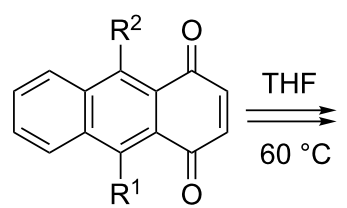

2c: $R^{1}=R^{2}=H$ d: $\mathrm{R}^{1}=\mathrm{Cl}, \mathrm{R}^{2}=\mathrm{OH}$<smiles>[Y10]C1=CC([Al])C2=C(S1)C(=O)c1c(c([R])c3ccccc3c1[R])C2=O</smiles>

$4 e-I(58-96 \%)$<smiles></smiles>

$5 a-d$

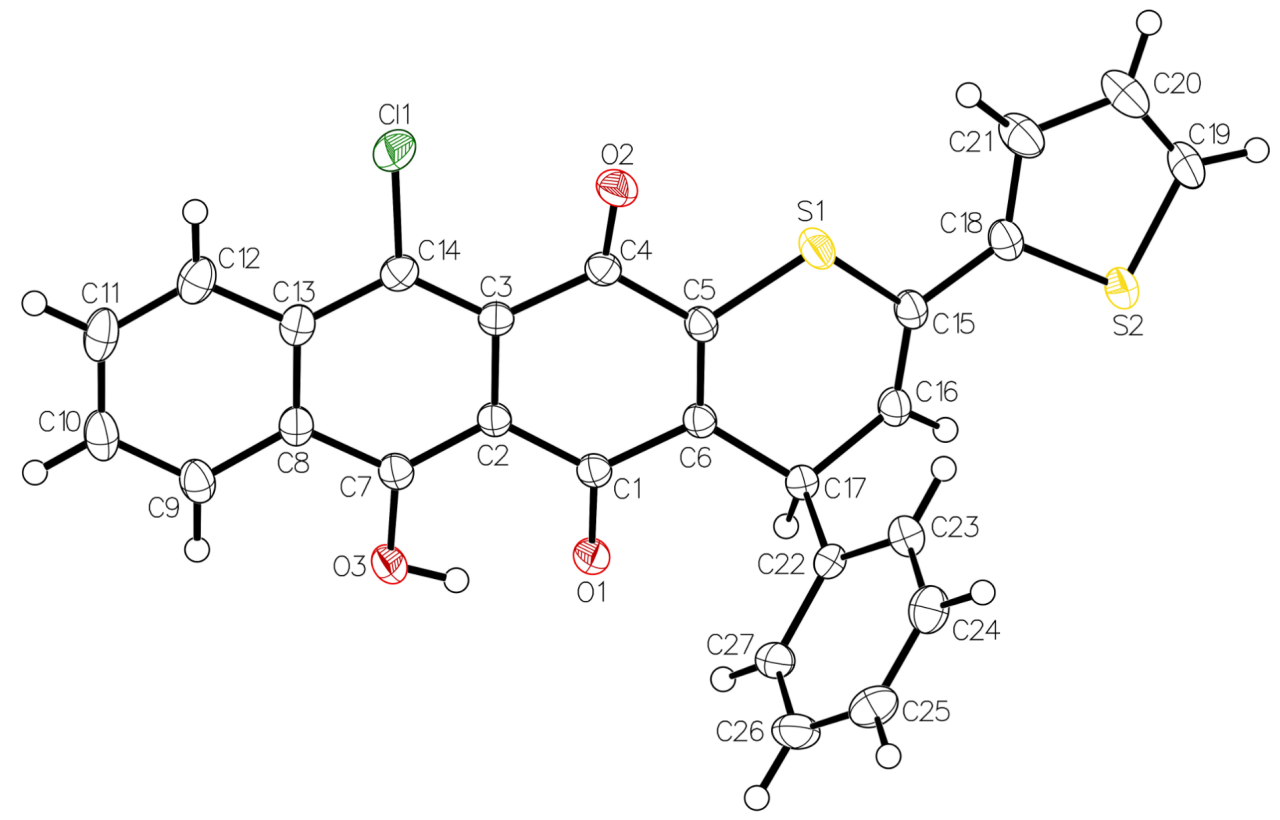

Figure 1: ORTEP plot [29] of the molecular structure of $\mathbf{4 k}$ showing the major conformation of the disordered thiophene ring $(50 \%$ probability ellipsoids; arbitrary numbering of the atoms).

The crystal structure of $\mathbf{4 k}$ is that of the regioisomer proposed on the basis of reactivity considerations. Since the space group is centrosymmetric, the compound in the crystal is racemic. The S-atom of the thiophene ring is disordered over two unequally occupied positions as a result of slight but opposite directions of envelope puckering of the ring. The hydroxy group forms an intramolecular hydrogen bond with the adjacent quinoid O-atom.

In addition to anthraquinones $\mathbf{2 b}$ and $\mathbf{2 c}$, the simple 1,4-benzoquinone (2a) was also tested in the reaction with thiochalcones 1. The reactions performed with $\mathbf{1 a}$ and $\mathbf{1 b}$ delivered the ex- pected $4 H$-thiochromene-5,8-diones $\mathbf{4 m}, \mathbf{n}$, which were isolated in good yields using flash chromatography, but underwent decomposition under ambient conditions, and none of them could be obtained in analytically pure form (Figure 2).

Vitamin $\mathrm{K}_{3}$ (2e, menadione), which is an important representative of 1,4-naphtoquinones, was also involved in the present study and tested in the reaction with diphenylthiochalcone (1a). In that case, however, a longer reaction time was required, and substantial amounts of decomposition products were formed. The chromatographic workup led to a brownish fraction, containing a single product, which was identified as the $[4+2]$ 
<smiles>O=C1C=CC(=O)C2=C1SC(c1ccccc1)=CC2c1ccccc1</smiles><smiles>O=C1C=CC(=O)C2=C1SC(c1ccccc1)=CC2c1cccs1</smiles>

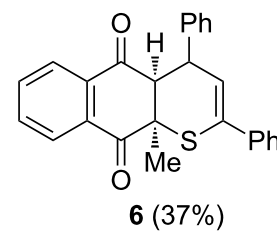

Figure 2: Products of the reactions of thiochalcones $\mathbf{1 a}$ and $\mathbf{1 b}$ with 1,4-benzoquinone (2a) and of $1 \mathbf{a}$ with menadione (2e).

cycloadduct 6 (Figure 2). Its structure was elucidated by ${ }^{1} \mathrm{H}$ NMR spectroscopy, which evidenced the presence of two doublets at $3.76(J=4.8 \mathrm{~Hz})$ and $6.50 \mathrm{ppm}(J=4.3 \mathrm{~Hz})$ and a triplet-like signal at $4.18 \mathrm{ppm}$ with $J \approx 4.5 \mathrm{~Hz}$. These signals were attributed to $\mathrm{HC}(4 \mathrm{a}), \mathrm{HC}(3)$ and $\mathrm{HC}(4)$, respectively. All attempts to prepare an analytically pure sample were unsuccessful. In contrast to all other initially formed cycloadducts, further oxidative conversion to the quinone structure was not possible in this case.

\section{Conclusion}

The present study demonstrates that 1,4-quinones are prone dienophiles in reactions with aryl/hetarylthiochalcones. These thia-Diels-Alder reactions led to a new class of $4 H$-thiopyran derivatives. The presence of a quinone system is an important structural aspect of this class as it is common in many naturally occurring compounds, e.g., dyes such as alizarin, carminic acid, and isoprenoid dyes, as well as drugs such as doxorubicin. In addition, the presence of thiophenyl substituents may be of importance for their applications in materials chemistry.

\section{Experimental}

General information: Solvents and chemicals were purchased and used as received without further purification. Products were purified by standard column chromatography on silica gel (230-400 mesh, Merck). Unless stated otherwise, yields refer to analytically pure samples. NMR spectra were recorded with a Bruker Avance III $600 \mathrm{MHz}$ instrument ( ${ }^{1} \mathrm{H}$ NMR: $600 \mathrm{MHz}$; ${ }^{13} \mathrm{C}$ NMR: $\left.151 \mathrm{MHz}\right)$. Chemical shifts are reported relative to solvent residual peaks $\left({ }^{1} \mathrm{H} \mathrm{NMR}: \delta=7.26 \mathrm{ppm}\left[\mathrm{CHCl}_{3}\right]\right.$; ${ }^{13} \mathrm{C}$ NMR: $\left.\delta=77.0 \mathrm{ppm}\left[\mathrm{CDCl}_{3}\right]\right)$. IR spectra were registered with a FTIR NEXUS spectrometer (as film or $\mathrm{KBr}$ pellets). UV-vis spectra were recorded using a UV-vis JASCO V-630 spectrophotometer. Melting points were determined in capillaries with a Stuart SMP30 apparatus with automatic temperature monitoring.

Starting materials: 1,4-Benzoquinone (2a) and 1,4-naphthoquinone (2b) were commercial reagents and used without further purification. 1,4-Anthraquinone (2c) was prepared from commercial quinizarine by treatment with sodium borohydride according to a literature procedure [27]. 5-Chloro-10-hydroxy1,4-anthraquinone (2d) was obtained by treatment of quinizarine with thionyl chloride according to the protocol described in [27]. Thiochalcones 1a-d were prepared according to our protocol reported in an earlier publication [16].

General procedure: A solution of $1 \mathrm{mmol}$ of the corresponding thiochalcone 1 and $1 \mathrm{mmol}$ of the 1,4-quinone 2 in $1 \mathrm{~mL}$ of dry THF was placed in a thick-walled glass tube, which was closed with a screw cap. The mixtures were heated at $60{ }^{\circ} \mathrm{C}$ for $2 \mathrm{~h}$ (for $\mathbf{4 a - n}$ ) or $48 \mathrm{~h}$ (for $\mathbf{6}$ ). In the last case, progress of the reaction was monitored by TLC, and an additional amount of thiochalcone 1a was added in small portions until menadione (2e) was completely consumed. The solvent was evaporated in vacuo and the crude mixtures were analyzed by ${ }^{1} \mathrm{H}$ NMR first and subsequently purified by flash chromatography using dichloromethane (for $\mathbf{4 a - n}$ ) or a mixture of petroleum ether and dichloromethane 1:1 (for 6) as the eluents. For products $\mathbf{4 a}-\mathbf{l}$, analytically pure samples were obtained by crystallization from petroleum ether with a small amount of dichloromethane. All attempts to obtain analytically pure samples for $4 \mathrm{~m}, \mathbf{4 n}$ and 6 were unsuccessful and the purification procedure led to formation of some decomposition products.

2,4-Diphenyl-4H-benzo $[g]$ thiochromene-5,10-dione (4a): Yield: $340 \mathrm{mg}$ (89\%). Red-orange crystals; $\mathrm{mp} 165^{\circ} \mathrm{C}$ (dec.); ${ }^{1} \mathrm{H}$ NMR $\delta 5.46\left(\mathrm{~d}, J_{\mathrm{H}, \mathrm{H}}=6.4 \mathrm{~Hz}, \mathrm{Ph}-\mathrm{CH}\right), 6.35(\mathrm{~d}$, $\left.J_{\mathrm{H}, \mathrm{H}}=6.4 \mathrm{~Hz}, \mathrm{C}=\mathrm{CH}\right), 7.24-7.25\left(\mathrm{~m}, 1 \mathrm{CH}_{\text {arom }}\right), 7.32-7.34(\mathrm{~m}$, $\left.2 \mathrm{CH}_{\text {arom }}\right), 7.41-7.43\left(\mathrm{~m}, 3 \mathrm{CH}_{\text {arom }}\right), 7.52-7.54\left(\mathrm{~m}, 2 \mathrm{CH}_{\text {arom }}\right)$, 7.61-7.62 (m, 2CH $\left.\mathrm{CH}_{\text {arom }}\right), 7.70-7.74\left(\mathrm{~m}, 2 \mathrm{CH}_{\text {arom }}\right), 8.10$ (dd, $\left.J_{\mathrm{H}, \mathrm{H}}=7.4 \mathrm{~Hz}, J_{\mathrm{H}, \mathrm{H}}=1.2 \mathrm{~Hz}, 1 \mathrm{CH}_{\mathrm{arom}}\right), 8.13(\mathrm{dd}$, $\left.J_{\mathrm{H}, \mathrm{H}}=7.4 \mathrm{~Hz}, J_{\mathrm{H}, \mathrm{H}}=1.2 \mathrm{~Hz}, 1 \mathrm{CH}_{\text {arom }}\right) \mathrm{ppm} ;{ }^{13} \mathrm{C} \mathrm{NMR} \delta 40.9$ (Ph-CH), 121.0, 126.6, 126.7, 127.0, 127.5, 128.4, 128.8, 128.9, $129.0,133.4,134.3\left(14 \mathrm{CH}_{\text {arom }}, \mathrm{C}=\mathrm{CH}\right), 131.7,132.0,132.2$, $136.4,137.4,142.3,144.54\left(6 \mathrm{C}_{\text {arom }}, C=\mathrm{CH}\right), 180.8,181.4$ $(2 \mathrm{C}=\mathrm{O})$ ppm; IR v: $3060(\mathrm{w}), 3028(\mathrm{w}), 1653$ (vs, 2C=O), 1591 (s), 1562 (m), 1489 (m), 1451 (m), 1334 (m), 1288 (vs), 1152 (m), $1106(\mathrm{w}), 1030$ (m), 913 (m), 834 (m), 710 (s), 694 (s) $\mathrm{cm}^{-1}$; UV-vis $\left(\mathrm{CH}_{2} \mathrm{Cl}_{2}\right) \lambda_{\text {max }} / \mathrm{nm}$ (lg \&): 243 (4.49), 332 (3.57), 478 (328); anal. calcd for $\mathrm{C}_{25} \mathrm{H}_{16} \mathrm{O}_{2} \mathrm{~S}$ (380.46): C, 78.92; $\mathrm{H}$, 4.24; S, 8.43; found: C, 78.87; H, 4.24; S, 8.37.

\section{2-Phenyl-4-(thiophen-2-yl)-4H-benzo[g]thiochromene-5,10-}

dione (4b): Yield: $365 \mathrm{mg}$ (94\%). Red-orange crystals; mp $168{ }^{\circ} \mathrm{C}$ (dec.); ${ }^{1} \mathrm{H}$ NMR $\delta 5.80\left(\mathrm{~d}, J_{\mathrm{H}, \mathrm{H}}=6.7 \mathrm{~Hz}\right.$, thiophen2-yl-CH), $6.40\left(\mathrm{~d}, J_{\mathrm{H}, \mathrm{H}}=6.7 \mathrm{~Hz}, \mathrm{C}=\mathrm{C} H\right), 6.95(\mathrm{dd}$, $\left.J_{\mathrm{H}, \mathrm{H}}=5.0 \mathrm{~Hz}, J_{\mathrm{H}, \mathrm{H}}=3.7 \mathrm{~Hz}, 1 \mathrm{CH}_{\text {arom }}\right), 7.06\left(\mathrm{~d}, J_{\mathrm{H}, \mathrm{H}}=3.5 \mathrm{~Hz}\right.$, $\left.1 \mathrm{CH}_{\text {arom }}\right), 7.19\left(\mathrm{dd}, J_{\mathrm{H}, \mathrm{H}}=5.0 \mathrm{~Hz}, J_{\mathrm{H}, \mathrm{H}}=1.0 \mathrm{~Hz}, 1 \mathrm{CH}_{\text {arom }}\right)$, 7.42-7.46 (m, 3CH $\left.\mathrm{CH}_{\text {arom }}\right), 7.63-7.65\left(\mathrm{~m}, 2 \mathrm{CH}_{\text {arom }}\right), 7.71-7.78$ $\left(\mathrm{m}, 2 \mathrm{CH}_{\text {arom }}\right), 8.13\left(\mathrm{dd}, J_{\mathrm{H}, \mathrm{H}}=7.6 \mathrm{~Hz}, J_{\mathrm{H}, \mathrm{H}}=1.1 \mathrm{~Hz}\right.$, 
$\left.1 \mathrm{CH}_{\text {arom }}\right), 8.18\left(\mathrm{dd}, J_{\mathrm{H}, \mathrm{H}}=7.6 \mathrm{~Hz}, J_{\mathrm{H}, \mathrm{H}}=1.1 \mathrm{~Hz}, 1 \mathrm{CH}_{\text {arom }}\right)$ ppm; ${ }^{13} \mathrm{C}$ NMR $\delta 35.2$ (thiophen-2-yl-CH), 119.9, 125.3, 125.6, $126.7,127.0,127.1,127.2,128.8,129.2,133.5,134.4$ $\left(12 \mathrm{CH}_{\text {arom }}, \mathrm{C}=\mathrm{CH}\right), 128.5,131.8,132.1,135.3,137.2,144.2$, $144.3\left(6 \mathrm{C}_{\text {arom }}, C=\mathrm{CH}\right), 180.6,181.4(2 \mathrm{C}=\mathrm{O}) \mathrm{ppm}$; IR v: 3075 (w), $3031(\mathrm{w}), 1652(\mathrm{vs}, 2 \mathrm{C}=\mathrm{O}), 1589(\mathrm{~s}), 1571(\mathrm{~s}), 1489(\mathrm{~m})$, 1442 (m), 1420 (w), 1331 (m), 1289 (vs), 1220 (m), 1151 (m), $1103(\mathrm{w}), 1033(\mathrm{w}), 919(\mathrm{w}), 831$ (m), $767(\mathrm{~m}), 704$ (s), 695 (s) $\mathrm{cm}^{-1}$; UV-vis $\left(\mathrm{CH}_{2} \mathrm{Cl}_{2}\right) \lambda_{\max } / \mathrm{nm}(\lg \varepsilon): 244$ (4.50), 338 (3.59), 463 (3.34); anal. calcd for $\mathrm{C}_{23} \mathrm{H}_{14} \mathrm{O}_{2} \mathrm{~S}_{2}$ (386.49): C, 71.48; H, 3.65; S, 16.59; found: C, 71.48; H, 3.65; S 16.59 .

4-Phenyl-2-(thiophen-2-yl)-4H-benzo $[g]$ thiochromene-5,10dione (4c): Yield: $355 \mathrm{mg}(92 \%)$. Red-orange crystals; $\mathrm{mp} 158{ }^{\circ} \mathrm{C}$ (dec.); ${ }^{1} \mathrm{H}$ NMR $\delta 5.42\left(\mathrm{~d}, J_{\mathrm{H}, \mathrm{H}}=6.5 \mathrm{~Hz}, \mathrm{Ph}-\mathrm{C} H\right)$, $6.41\left(\mathrm{~d}, J_{\mathrm{H}, \mathrm{H}}=6.5 \mathrm{~Hz}, \mathrm{C}=\mathrm{C} H\right), 7.01\left(\mathrm{dd}, J_{\mathrm{H}, \mathrm{H}}=5.2 \mathrm{~Hz}\right.$, $\left.J_{\mathrm{H}, \mathrm{H}}=3.4 \mathrm{~Hz}, 1 \mathrm{CH}_{\text {arom }}\right), 7.23-7.25\left(\mathrm{~m}, 1 \mathrm{CH}_{\text {arom }}\right), 7.28-7.33$ $\left(\mathrm{m}, 3 \mathrm{CH}_{\text {arom }}\right), 7.36\left(\mathrm{dd}, J_{\mathrm{H}, \mathrm{H}}=3.4 \mathrm{~Hz}, J_{\mathrm{H}, \mathrm{H}}=1.0 \mathrm{~Hz}\right.$, $\left.1 \mathrm{CH}_{\text {arom }}\right), 7.50-7.51\left(\mathrm{~m}, 2 \mathrm{CH}_{\text {arom }}\right), 7.69-7.75\left(\mathrm{~m}, 2 \mathrm{CH}_{\text {arom }}\right)$, $8.09\left(\mathrm{dd}, J_{\mathrm{H}, \mathrm{H}}=7.4 \mathrm{~Hz}, J_{\mathrm{H}, \mathrm{H}}=1.3 \mathrm{~Hz}, 1 \mathrm{CH}_{\mathrm{arom}}\right), 8.14(\mathrm{dd}$, $\left.J_{\mathrm{H}, \mathrm{H}}=7.4 \mathrm{~Hz}, J_{\mathrm{H}, \mathrm{H}}=1.2 \mathrm{~Hz}, 1 \mathrm{CH}_{\text {arom }}\right) \mathrm{ppm} ;{ }^{13} \mathrm{C}$ NMR $\delta 40.6$ (Ph- $\mathrm{CH}), 119.9,125.3,125.7,126.6,127.0,127.6,127.7,128.4$, $129.0,133.4,134.2\left(12 \mathrm{CH}_{\text {arom }}, \mathrm{C}=\mathrm{CH}\right), 125.9,131.7,132.1$, $136.8,140.3,141.8,143.9\left(6 \mathrm{C}_{\text {arom }}, C=\mathrm{CH}\right), 180.6,181.3$ $(2 \mathrm{C}=\mathrm{O})$ ppm; IR v: $3085(\mathrm{w}), 3063(\mathrm{w}), 1652(\mathrm{vs}, 2 \mathrm{C}=\mathrm{O}), 1590$ (s), $1573(\mathrm{~s}), 1490(\mathrm{~m}), 1453(\mathrm{~m}), 1347(\mathrm{~m}), 1335(\mathrm{~m}), 1289$ (vs), $1230(\mathrm{~m}), 1150(\mathrm{~m}), 1111(\mathrm{w}), 1074(\mathrm{~m}), 839(\mathrm{~m}), 880$ (m), $815(\mathrm{~m}), 710(\mathrm{~s}), 696(\mathrm{~s}) \mathrm{cm}^{-1} ; \mathrm{UV}-\mathrm{vis}\left(\mathrm{CH}_{2} \mathrm{Cl}_{2}\right) \lambda_{\max } / \mathrm{nm}$ (lg ع): 269 (4.42), 473 (3.12); anal. calcd for $\mathrm{C}_{23} \mathrm{H}_{14} \mathrm{O}_{2} \mathrm{~S}_{2}$ (386.49): C, 71.48; H, 3.65; S, 16.59; found: C, 71.48; H, 3.70; S, 16.58 .

11-Chloro-6-hydroxy-4-phenyl-2-(thiophen-2-yl)-4Hnaphtho[2,3-g]thiochromene-5,12-dione (4k): Yield: $285 \mathrm{mg}$ (59\%). Dark red crystals; mp $162{ }^{\circ} \mathrm{C}\left(\mathrm{dec}\right.$.); ${ }^{1} \mathrm{H}$ NMR $\delta 5.78(\mathrm{~d}$, $J_{\mathrm{H}, \mathrm{H}}=6.7 \mathrm{~Hz}$, thiophen-2-yl-CH), $6.48\left(\mathrm{~d}, J_{\mathrm{H}, \mathrm{H}}=6.8 \mathrm{~Hz}\right.$, $\mathrm{C}=\mathrm{C} H), 6.96\left(\mathrm{dd}, J_{\mathrm{H}, \mathrm{H}}=4.9 \mathrm{~Hz}, J_{\mathrm{H}, \mathrm{H}}=3.8 \mathrm{~Hz}, 1 \mathrm{CH}_{\text {arom }}\right)$, $7.01-7.11\left(\mathrm{~m}, 2 \mathrm{CH}_{\text {arom }}\right), 7.21\left(\mathrm{dd}, J_{\mathrm{H}, \mathrm{H}}=4.9 \mathrm{~Hz}\right.$, $\left.J_{\mathrm{H}, \mathrm{H}}=1.0 \mathrm{~Hz}, 1 \mathrm{CH}_{\text {arom }}\right), 7.35\left(\mathrm{dd}, J_{\mathrm{H}, \mathrm{H}}=4.9 \mathrm{~Hz}\right.$, $\left.J_{\mathrm{H}, \mathrm{H}}=1.0 \mathrm{~Hz}, 1 \mathrm{CH}_{\text {arom }}\right), 7.40\left(\mathrm{dd}, J_{\mathrm{H}, \mathrm{H}}=4.9 \mathrm{~Hz}\right.$, $\left.J_{\mathrm{H}, \mathrm{H}}=1.0 \mathrm{~Hz}, 1 \mathrm{CH}_{\text {arom }}\right), 7.76-7.80\left(\mathrm{~m}, 1 \mathrm{CH}_{\text {arom }}\right), 7.87-7.89$ $\left(\mathrm{m}, 1 \mathrm{CH}_{\text {arom }}\right), 8.59\left(\mathrm{~d}, J_{\mathrm{H}, \mathrm{H}}=8.2 \mathrm{~Hz}, 1 \mathrm{CH}_{\text {arom }}\right), 8.63(\mathrm{~d}$, $\left.J_{\mathrm{H}, \mathrm{H}}=8.2 \mathrm{~Hz}, 1 \mathrm{CH}_{\text {arom }}\right), 14.98(\mathrm{~s}, \mathrm{OH}) \mathrm{ppm} ;{ }^{13} \mathrm{C}$ NMR $\delta 34.5$ (thiophen-2-yl- $\mathrm{CH}), 118.7,125.0,125.5,125.7,125.8,126.2$, $127.2,127.5,127.8,130.1,132.2\left(10 \mathrm{CH}_{\text {arom }}, \mathrm{C}=\mathrm{CH}\right), 108.8$, $122.5,127.6,128.5,134.8,135.2,139.9,143.5,142.1,147.4$, $162.6\left(10 \mathrm{C}_{\text {arom }}, C=\mathrm{CH}\right), 179.0,184.0(2 \mathrm{C}=\mathrm{O}) \mathrm{ppm}$; IR v: 3022 (w), 2953 (s), 2923 (w), 1655 (s, 2C=O), 1601 (s), 1584 (s), 1565 (s), 1491 (m), 1427 (s), 1397 (s), 1360 (s), 1360 (m), 1333 (m), 1242 (vs), $1170(\mathrm{~m}), 902(\mathrm{~s}), 852(\mathrm{~m}), 832(\mathrm{~m}), 758(\mathrm{~m})$, 699 (vs) $\mathrm{cm}^{-1}$; UV-vis $\left(\mathrm{CH}_{2} \mathrm{Cl}_{2}\right) \lambda_{\max } / \mathrm{nm}(\mathrm{lg} \varepsilon): 245$ (4.69),
294 (4.32), 487 (3.91); anal. calcd for $\mathrm{C}_{27} \mathrm{H}_{15} \mathrm{ClO}_{3} \mathrm{~S}_{2}$ (486.99): C, 66.59; H, 3.10; S, 13.17; found: C, 66.39; H, 3.07; S, 13.06 .

\section{(4aSR,10a $R S)$-10a-Methyl-2,4-diphenyl-4H-benzo[g]thio-} chromene-5,10-(4aH,10aH)-dione (6): Yield: $145 \mathrm{mg}$ (37\%). Yellow-brown viscous oil; ${ }^{1} \mathrm{H}$ NMR $\delta 1.94\left(\mathrm{~s}, \mathrm{CH}_{3}\right), 3.76(\mathrm{~d}$, $\left.J_{\mathrm{H}, \mathrm{H}}=4.8 \mathrm{~Hz}, 1 \mathrm{CH}\right), 4.18\left(\mathrm{t}, J_{\mathrm{H}, \mathrm{H}}=4.5 \mathrm{~Hz}, 1 \mathrm{CH}\right), 6.50(\mathrm{~d}$, $\left.J_{\mathrm{H}, \mathrm{H}}=4.3 \mathrm{~Hz}, 1 \mathrm{CH}\right), 7.08-7.12(\mathrm{~m}, 1 \mathrm{CH}), 7.16-7.19(\mathrm{~m}, 2 \mathrm{CH})$, $7.30-7.38(\mathrm{~m}, 5 \mathrm{CH}), 7.54-7.57(\mathrm{~m}, 2 \mathrm{CH}), 7.58-7.61(\mathrm{~m}, 2 \mathrm{CH})$, 7.79-7.82 (m, 1CH), 7.91-7.93 (m, 1CH) ppm; ${ }^{13} \mathrm{C} \mathrm{NMR}$

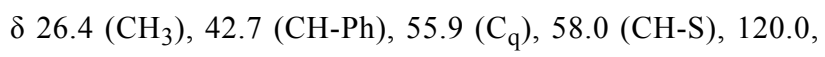
$126.3,126.8,126.9,127.3,128.0,128.5,128.6,129.5,133.8$, $134.2\left(14 \mathrm{CH}_{\text {arom }}, \mathrm{C}=\mathrm{CH}\right), 132.4,134.4,134.6,138.9,139.6$ $\left(4 \mathrm{C}_{\text {arom }}, C=\mathrm{CH}\right), 193.9,194.2(2 \mathrm{C}=\mathrm{O}) \mathrm{ppm}$; IR v: $3062(\mathrm{w})$, $3034(\mathrm{w}), 2957(\mathrm{w}), 1696(\mathrm{vs})$ and $1691(\mathrm{~s}, 2 \mathrm{C}=\mathrm{O}), 1614(\mathrm{~s})$, 1489 (m), 1451 (m), 1266 (s), 1013 (m), 979 (m), 761 (s), 692 (s) $\mathrm{cm}^{-1}$; ESIMS (for $\mathrm{C}_{26} \mathrm{H}_{20} \mathrm{O}_{2} \mathrm{~S}$ ): 395 (100, [M - 1] $]^{-}$).

\section{Supporting Information}

CCDC-1838975 contains the supplementary crystallographic data for this paper. These data can be obtainded free of charge from the Cambridge Crystallographic Data Centre via http://www.ccdc.cam.ac.uk/structures.

\section{Supporting Information File 1}

Experimental data for selected compounds 4, details of the crystal structure determination, and copies of ${ }^{1} \mathrm{H}$ and ${ }^{13} \mathrm{C}$ NMR spectra for all products.

[https://www.beilstein-journals.org/bjoc/content/ supplementary/1860-5397-14-156-S1.pdf]

\section{Acknowledgements}

Financial support by the National Science Center (NCN, Poland) in the framework of the Maestro-3 grant (2012/06A/ ST5/00219) is gratefully acknowledged.

\section{ORCID ${ }^{\circledR}$ iDs}

Grzegorz Mlostoń - https://orcid.org/0000-0003-3631-3239 Katarzyna Urbaniak - https://orcid.org/0000-0002-1633-2750 Paweł Urbaniak - https://orcid.org/0000-0003-1458-7765 Anthony Linden - https://orcid.org/0000-0002-9343-9180

\section{References}

1. Boger, D. L.; Weinreb, S. N., Eds. Hetero Diels-Alder Methodology in Organic Synthesis; Organic Chemistry, A Series of Monographs; Academic Press Inc.: London, 1987; Vol. 47.

2. Blond, G.; Gulea, M.; Mamane, V. Curr. Org. Chem. 2016, 20 , 2161-2210. doi:10.2174/1385272820666160216000401 
3. Schmidt, R. R. Acc. Chem. Res. 1986, 19, 250-259. doi:10.1021/ar00128a004

4. Heravi, M. M.; Ahmadi, T.; Ghavidel, M.; Heidari, D.; Hamidi, H. RSC Adv. 2015, 5, 101999-102075. doi:10.1039/C5RA17488K

5. Gregoritza, M.; Brandl, F. P. Eur. J. Pharm. Biopharm. 2015, 97, 438-453. doi:10.1016/j.ejpb.2015.06.007

6. Constantino, A. F.; Francisco, C. S.; Cubides-Roman, D. C.; Lacerda, V., Jr. Curr. Org. Synth. 2018, 15, 84-104. doi:10.2174/1570179414666170517170230

7. Funel, J.-A.; Abele, S. Angew. Chem., Int. Ed. 2013, 52, 3822-3863. doi:10.1002/anie.201201636

8. Cui, H.-L.; Chouthaiwale, P. V.; Yin, F.; Tanaka, F. Org. Biomol. Chem. 2016, 14, 1777-1783. doi:10.1039/c5ob02393a

9. Liu, L.; Kim, H.; Xie, Y.; Farès, C.; Kaib, P. S. J.; Goddard, R.; List, B. J. Am. Chem. Soc. 2017, 139, 13656-13659. doi:10.1021/jacs.7b08357

10. Hejmanowska, J.; Jasiński, M.; Mlostoń, G.; Albrecht, $七$. Eur. J. Org. Chem. 2017, 950-954. doi:10.1002/ejoc.201601307

11. Breu, J.; Höcht, P.; Rohr, U.; Schatz, J.; Sauer, J. Eur. J. Org. Chem. 1998, 2861-2873. doi:10.1002/(SICI)1099-0690(199812)1998:12<2861::AID-EJOC2861> 3.0.CO;2-C

12. Rohr, U.; Schatz, J.; Sauer, J. Eur. J. Org. Chem. 1998, 2875-2883. doi:10.1002/(SICI)1099-0690(199812)1998:12<2875::AID-EJOC2875> 3.0.CO;2-N

13. Wilker, S.; Erker, G. J. Am. Chem. Soc. 1995, 117, 10922-10930. doi:10.1021/ja00149a015

14. Mlostoń, G.; Grzelak, P.; Linden, A.; Heimgartner, $H$. Chem. Heterocycl. Compd. 2017, 53, 518-525. doi:10.1007/s10593-017-2086-9

15. Grzelak, P.; Utecht, G.; Jasiński, M.; Mlostoń, G. Synthesis 2017, 49, 2129-2137. doi:10.1055/s-0036-1588774

16. Mlostoń, G.; Grzelak, P.; Heimgartner, H. J. Sulfur Chem. 2017, 38 , 1-10. doi:10.1080/17415993.2016.1230857

17. Karakasa, T.; Yamaguchi, H.; Motoki, S. J. Org. Chem. 1980, 45, 927-930. doi:10.1021/jo01294a001

18. Saito, T.; Fujii, H.; Hayashibe, S.; Matsushita, T.; Kato, H.; Kobayashi, K. J. Chem. Soc., Perkin Trans. 1 1996, 1897-1903. doi:10.1039/P19960001897

19. Hejmanowska, J.; Jasiński, M.; Wojciechowski, J.; Mlostoń, G.; Albrecht, Ł. Chem. Commun. 2017, 53, 11472-11475. doi:10.1039/C7CC06518C

20. Mlostoń, G.; Hamera-Fałdyga, R.; Heimgartner, H. J. Sulfur Chem. 2018, 39, 322-331. doi:10.1080/17415993.2018.1427749

21. Stegmann, W.; Uebelhart, P.; Heimgartner, H. Helv. Chim. Acta 1983, 66, 2252-2268. doi:10.1002/hlca.19830660736

22. Collis, G. E.; Wege, D. Aust. J. Chem. 2003, 56, 903-908. doi:10.1071/CH03060

23. Hamadi, N. B.; Msaddek, M. Heterocycl. Commun. 2006, 12, 457-462. doi:10.1515/HC.2006.12.6.457

24. Laatsch, H. Z. Naturforsch. 1984, 39b, 244-247.

25. Kotera, M.; Lehn, J.-M.; Vigneron, J.-P. Tetrahedron 1995, 51 , 1953-1972. doi:10.1016/0040-4020(94)01076-C

26. Kotha, S.; Manivannan, E. Indian J. Chem., Sect. B 2002, 41, 808-811.

27. Flock, M.; Nieger, M.; Breitmaier, E. Liebigs Ann. Chem. 1993, 451-455. doi:10.1002/jlac.199319930175

28. Nor, S. M. M.; Sukari, M. A. H. M.; Azziz, S. S. S. A.; Fah, W. C.; Alimon, H.; Juhan, S. F. Molecules 2013, 18, 8046-8062. doi: $10.3390 /$ molecules 18078046
29. Johnson, C. K. ORTEP II, Report ORNL-5138, Oak Ridge National Laboratory, Oak Ridge, Tennessee, 1976.

\section{License and Terms}

This is an Open Access article under the terms of the Creative Commons Attribution License (http://creativecommons.org/licenses/by/4.0). Please note that the reuse, redistribution and reproduction in particular requires that the authors and source are credited.

The license is subject to the Beilstein Journal of Organic Chemistry terms and conditions:

(https://www.beilstein-journals.org/bjoc)

The definitive version of this article is the electronic one which can be found at:

doi:10.3762/bjoc. 14.156 